\title{
Kernos
}

Revue internationale et pluridisciplinaire de religion grecque antique

16 | 2003

Varia

\section{Les Tragiques Grecs. Eschyle - Sophocle - Euripide}

\section{Alain Moreau}

\section{OpenEdition}

\section{Journals}

Édition électronique

URL : http://journals.openedition.org/kernos/844

DOI : $10.4000 /$ kernos.844

ISSN : 2034-7871

\section{Éditeur}

Centre international d'étude de la religion grecque antique

\section{Édition imprimée}

Date de publication : 1 janvier 2003

Pagination : 369-372

ISSN : 0776-3824

\section{Référence électronique}

Alain Moreau, "Les Tragiques Grecs. Eschyle - Sophocle - Euripide », Kernos [En ligne], 16 | 2003, mis en ligne le 14 avril 2011, consulté le 21 septembre 2020. URL : http://journals.openedition.org/kernos/ 844 ; DOI : https://doi.org/10.4000/kernos.844 
reste pas dit que ces deux caractérisations s'excluent mutuellement; elles correspondent peut-être à une évolution diachronique de l'école pythagoricienne.

Dans cette seconde section, on peut épingler le copieux essai d'A. Mele sur Megale Hellas e pitagorismo (aux p. 297-333) qui retiendra l'attention des historiens de la GrandeGrèce. Mele s'efforce en effet de repréciser le concept de Megale Hellas, en Italie méridionale et en Sicile, par le biais d'un dossier textuel extrêmement riche et détaillé, et de préciser le rapport avec le pythagorisme: "il pitagorismo non solo dispiegò la sua attività nella Megale Hellas e si giovò della sua prosperità, ma se ne fece anche valorizzatore e diffusore » (p. 333).

Mais le pythgorisme fut aussi romain, comme nous le rappelle la belle contribution d'A. Storchi Marino: Il pitagorismo romano. Per un bilancio di studi recenti, une étude qui fait une large place à l'historiographie, mais affronte aussi la question des disciples romains de Pythagore, de la mise en parallèle avec Numa, de la prétendue citoyenneté romaine de Pythagore, etc.

On pourra regretter deux choses: tout d'abord que le volume ou, mieux encore, que chaque partie ne se termine pas par une conclusion. Le chantier mis en cuvre par nos collègues napolitains est remarquable, multiforme, très enrichissant, mais on aurait aimé que l'un d'entre eux prenne le soin de dresser un bilan des acquis ou des pistes au terme du travail, ne serait-ce que parce que la «Presentazione » occupe tout au plus une demipage et n'apprend pratiquement rien des enjeux réels des problématiques. Ensuite, des indices eussent été très utiles, quoiqu'ils se fassent rares dans les volumes collectifs.

Cela dit, la qualité du volume est telle qu'il servira assurément de point de référence en la matière. Il fournit au suprplus une bibliographie très fournie. On signalera enfin aux lecteurs la récente parution, en deux volumes, d'un recueil des textes relatifs aux religions à mystères : Paolo Scarpi, Le religioni dei misteri, Milan 2002, les textes orphiques figurant dans le premier tome (texte grec, traduction italienne, commentaire; $c f$. ici-même le compte rendu d'André Motte).

Corinne Bonnet

(Université de Toulouse-Le Mirail)

Les Tragiques Grecs. Escbyle - Sophocle - Euripide. Théâtre complet avec un choix de fragments. Traduction nouvelle, notices et notes de Victor-Henri Debidour. Édité avec une introduction générale et un dossier sur la tragédie par Paul Demont et Anne Lebeau, Paris, Éd. de Fallois, Le Livre de Poche, 1999. 1 vol. $11 \times 18 \mathrm{~cm}, 1998$ p. (La Pochothèque). ISBN : 2-253-13246-2.

Les Tragiques grecs, Eschyle - Sophocle - Euripide, édition établie par Louis Bardollet, Bernard Deforge et Jules Villemonteix; ouvrage dirigé par Bernard Deforge et François Jouan, Paris, Robert Laffont, 2001. 2 vols 13, $5 \times 19,5 \mathrm{~cm}, 864$ et $832 \mathrm{p}$. (Collection Bouquins). ISBN : 2-221-06553-0 \& 2-221-06554-9.

Quelle bonne nouvelle! Les Tragiques grecs sont un « créneau porteur »! En deux ans deux traductions avec un dossier abondant et sérieux. Dans les Cabier's du GITA 14 (2001), j'ai déjà eu l'occasion de dire tout le bien que je pensais de l'ouvrage de 1999. La traduction de la Pochothèque est l'œuvre de Victor-Henri Debidour, dont on apprécie depuis longtemps les savoureuses traductions d'Aristophane. On reconnaît immédiatement une traduction de V.H. Debidour. C'est un amoureux de la langue française, surtout de la langue française dans son enfance et son adolescence. Ses lectures préférées vont de la Chanson de Roland à Rabelais. Il adore cette langue pittoresque, colorée, charnue, qui n'est pas encore passée sous les fourches caudines du Samnite Vaugelas. Qu'on en juge avec le début de sa traduction des Perses: l'aboi, l'ost, les mariniers, les paluds, la charrerie, l'arroi, l'estoc, le bâti, le planchéiage, la guivre... Le Servateur au vers 8 des Sept contre Thèbes, c'est chez Rabelais qu'il faut aller le chercher et mon ordinateur, un puriste, souligne ce mot abominable d'un trait rouge ondulé qui marque la violence de son indignation. Et que dire de "l'épouse partie, affriandée d'un homme » au vers 411 de l'Agamemnon, premier stasimon! Il faut aussi remonter jusqu'au xive siècle pour en trouver la trace... Mais le traducteur sait raison garder et adapter la langue à l'auteur qu'il 
traduit. Puisque Eschyle incarne la jeunesse de la tragédie, Sophocle incarnera la maturité : si au vers 1289 de l'Ajax on trouve un «barbaresque » qui remonte à 1534, la fréquence des mots anciens a notablement diminué. Il n'empêche que dans Ipłigénie à Aulis, la clemière des tragédies d'Euripide, on découvre un "fief » au vers 91 qui fleure bon son Moyen Âge.

Le dossier est établi, avec leur compétence habituelle, par Anne Lebeau et Paul Demont. L'introduction générale est l'œuvre d'Anne Lebeau : origines du théâtre, particularités de la tragédie, biographie des poètes tragiques, tableau chronologique. Chaque pièce est précédée d'une introduction et d'une analyse. Les notes sont de V.H. Debidour (informations et attention attirée sur le choix de telle leçon ou de telle conjecture quand il ne suit pas les choix de Mazon). Après les tragédies conservées est donnée la traduction d'un choix de fragments, non seulement des trois grands tragiques, mais aussi d'auteurs dont il ne nous reste que des bribes, comme Théodecte, Agathon, Carcinos, Iophon, Ion, Chérémon, sans compter des adespota, les fragments sans maitre. À la fin de l'ouvrage on trouve une bibliographie : introductions générales au théâtre grec antique, à la tragédie, études sur Eschyle, Sophocle, Euripide, et sur chacune de leurs pièces, un utile lexique et un index des noms propres qui permet de retrouver facilement les occurrences dans l'ouvrage. Un travail très solide et qui rendra de grands services.

Les deux volumes publiés dans la collection «Bouquins» présentent des qualités équivalentes. L'introduction générale, par Bernard Deforge et François Jouan, est intitulée Du rituel au spectacle. Naissance et développement de la tragédie grecque. Elle comporte quatre parties : 1. Autour de la tragédie grecque. 2. La tragédie antique. Vie d'un genre littéraire. 3. La représentation théâtrale. 4. La mort sur la scène tragique. Rituel et spectacle (un sujet cher à Bernard Deforge, auteur d'un ouvrage intitulé Le Festival des cadavres. Morts et mises à mort dans la tragédie grecque, Paris, Les Belles Lettres, 1997). L'introduction est suivie d'un glossaire utile, d'une Cbronologie des vie et ve siècles grecs. Tragédies et événements et de Repères bibliograpbiques sur la tragédie grecque en général.

Vient ensuite le travail sur Eschyle. Traduction de Louis Bardollet et Bernard Deforge, à partir d'une traduction publiée en 1975 (Belles Lettres/Denoël), mais entièrement revue et corrigée; notice de B. Deforge; présentations et notes de L. Bardollet et B. Deforge. La notice donne tout d'abord la traduction de la Vie d'Eschyle faite par André Wartelle; une chronologie eschyléenne; un développement sur le texte, œuvre perdue et ouvre conservée; la transmission; un tableau d'ensemble de cette œuvre avec un commentaire sur le sujet; une étude sur le "créateur de la tragédie "; des repères bibliographiques limités à l'œuvre d'Eschyle en général. C'est le seul point faible du livre par rapport au DebidourLebeau-Demont : ceux-ci donnent également les principales éditions et commentaires des pièces. En revanche, divine surprise ou plutôt rencontre inévitable, les traducteurs sont en plein accord avec les choix opérés par V.H. Debidour et revendiquent les audaces de leur traduction. Le style d'Eschyle et sa pensée n'ont rien de classique : "traduire Eschyle de la même manière que Sophocle... est aussi déplacé que de vouloir traduire semblablement Rabelais, par exemple, et nos grands auteurs du XviI ${ }^{e}$ siècle ». La communauté de vue avec V.H. Debidour est admirable. Qu'on en juge : "Nous ne nous sommes pas interdit l'usage de mots rares ou inusités aujourd'hui, voire de néologismes ( se douloir" pour «se plaindre »; « la bastringue » pour « le bastingage; «poindre » au sens transitif de «piquer », "blesser »; " pointure " pour " piqûre »; " racquérir »; " glatir »... (mon ordinateur puriste s'étrangle de fureur). Je n'irai pas jusqu'à dire qu'il n'y a rien de classique dans le style et la pensée d'Eschyle, car je le crois tout de même plus proche de Sophocle que de Thespis et Pratinas, mais je pense qu'il y a cle la jeunesse, de la verdeur et de la violence dans son œuvre, parce qu'il participe à la fois à la naissance d'un nouvel art et à la naissance, nécessairement convulsive, d'un nouveau monde, celui de la démocratie (voir la fin de l'Orestie), qui succède à la tyrannie (voir le comportement de Zeus dans Prométhée enchaîné). Suivent la présentation et la traduction des sept pièces du dramaturge qui applique les principes énoncés dans l'introduction. Chaque fois que la tragédie a suscité d'autres pièces sur le même sujet (jusqu'à notre époque), elles sont indiquées à la fin de l'introduction. Cette règle vaut pour les trois auteurs. Évidemment, comme la liste est incommensurable, on peut toujours prendre un malin plaisir à souligner les manques. Personnellement j’aime beaucoup la Médée de Max Rouquette (version occitane, 1989, version française, 1992). 
C'est Jules Villemonteix qui présente Sophocle. Il commence par la traduction de la Vie qui figure en tête de onze manuscrits médiévaux des tragédies. Il en montre l'intérêt et les limites et dégage les principales caractéristiques de sa vie. Comme le dit Ion de Chios Sophocle était un «citoyen de l'élite », pleinement lié à la vie intellectuelle et politique de sa cité, très apprécié du public athénien puisqu'il débuta par une victoire en 468 av. J.-C. et triompha dix-huit fois (ce qui veut dire que soixante-douze pièces furent couronnées, puisque chaque fois il fallait présenter trois tragédies et un drame satyrique). Il prend volontiers ses sujets chez Homère ou dans les poèmes du cycle, même si les hasards de la transmission nous ont conservé trois pièces du cycle thébain. Ses héros sont des actifs, à forte personnalité, volontaires, passionnés, refusant de céder... et par conséquent destinés à la solitude et à la mort. Même si la dernière pièce conservée, Edipe à Colone, implique un certain apaisement (CEdipe étend sa protection sur l'Attique), la malédiction qu'arrivé au terme de se vie il lance contre son fils Polynice prouve qu'il reste fondamentalement le même, un violent, un solitaire, un indomptable : «Il reste une Érinys, plus redoutable que la vraie ». À la fin de l'introduction sont indiqués les ouvrages généraux les plus accessibles sur l'œuvre de Sophocle ainsi que les commentaires des pièces.

C'est la traduction de Mazon qui a été reprise. Sans doute J. Villemonteix aurait-il préféré fournir sa propre traduction, mais cela aurait retardé la publication. C'est pourquoi il s'est contenté d'aménager la traduction de la collection Budé, toujours judicieusement : ainsi le ptèxeian du vers 171 de l'Ajax que Mazon rendait par « ils se terreront» devient «ils se blottiront». En effet ce verbe est à mettre en rapport avec le nom ptax qui signifie la hase, littéralement « qui se blottit » (voir Eschyle, Agamemnon, 137) et ptôx, le lièvre (Iliade XVII, 676).

Pour chaque tragédie sont donnés l'argument (ou les arguments) de la pièce, la légende avec les sources, la date de la pièce et une analyse détaillée. Le volume s'achève par un index mythologique, historique et topographique qui concerne les trois Tragiques et que l'on retrouve à la fin du tome II.

Celui-ci est entièrement consacré à Euripide, dont le total des tragédies conservées est supérieur au total des tragédies conservées pour Eschyle et Sophocle réunis. Le maître d'œuvre est François Jouan. Selon le principe adopté pour les autres dramaturges, François Jouan rapporte la vie et l'œuvre d'Euripide d'après les témoignages de l'Antiquité, mais ces témoignages sont douteux et au total on sait fort peu de choses sur Euripide : «Les seuls repères chronologiques assurés de son existence sont liés à son ouvre tragique, ce qui confirme l'image d'un Euripide tout entier voué à la composition et à la représentation de ses drames ». Euripide empruntait ses sujets à des cycles légendaires très divers : guerre de Troie, Dionysos, Héraclès, légendes attiques, orientales, etc. L'on constate néanmoins que sur dix-huit pièces conservées quinze abordent des légendes déjà mises en scène par Eschyle, Sophocle ou d'autres dramaturges. Mais il les traite de façon très personnelle et diverse. Après l'examen du mythe, de l'intrigue et de la structure des tragédies d'Euripide, vient l'étude des personnages, de sa vision des dieux, des hommes et de leur mystère, du monde contemporain. Fr. Jouan souligne la richesse de sa langue, la beauté de sa poésie et remet en cause le mythe d'un dramaturge qui aurait été incompris de ses concitoyens. Il a eu des admirateurs fervents (les Athéniens ont pleuré au moment de sa mort) et au $\mathrm{F}^{\mathrm{e}}$ siècle sa popularité l'a emporté définitivement sur celle des autres poètes dramatiques. L'introduction se termine par des « repères bibliographiques », éditions et études générales sur Euripide.

Chaque pièce est précéclée d'une introduction qui résume l'intrigue et qui, par ailleurs, constitue une brève étude. En ce qui concerne la traduction, le même principe a été adopté que pour Sophocle : aménagement de la traduction de la collection Budé. Pour les Bacchantes Jean Irigoin a participé à la révision aux côtés de François Jouan. Prenons l'exemple d'Alceste. La cure de rajeunissement apparâ̂t dès le début : vers 12, «en leurrant les Parques » devient « en dupant les Moires »; vers 13, « qu'Admète échappât au trépas " clevient "qu'Admète échappât à Hadès " (puisque le nom du dieu est prononcé, il n'y a aucune raison de l'enlever); vers 24 , «Déjà voici le Trépas » devient «Déjà voici Thanatos » (Thanatos, frère d'Hypnos, représenté sous la forme d'un homme robuste, barbu, avec des ailes, a sa place dans le panthéon grec; il n'y a aucune raison de le métamorphoser en simple abstraction). À la fin du volume nous retrouvons le même index mythologique, historique et topographique qu'à la fin du tome I. 
Au lecteur de faire son choix entre les deux éditions. De toute façon il sera excellent.

\section{Alain Moreau \\ (Université de Montpellier)}

Pierre Voelke, Un théatre de la marge. Aspects figuratifs et configurationnels du. drame satyrique dans l'Athènes classique, Bari, Levante Editori, 2001. 1 vol. 15,5× $21 \mathrm{~cm}, 471$ p., 28 fig. sur planches ("Le Rane». Collana di Studi e Testi, Studi - 31). ISBN : 88-7949-267-5.

Cet ouvrage est issu d'une thèse de doctorat soutenue à l'Université de Lausanne en 1999 , mais la thèse est clevenue un vrai livre. Se mesurant à un corpus difficile, essentiellement fragmentaire, l'A. nous propose un parcours d'une grande clarté, servi par un style aussi élégant que limpide, et une parfaite maitrise de la documentation, qu'il s'agisse des textes anciens, systématiquement traduits, ou des travaux, dont la bibliographie atteste l'abondance et les notes infrapaginales, l'utilisation effective et pertinente.

Après une brève insertion du propos dans la diachronie - l'histoire présumée du genre satyrique - et dans la réflexion des modernes - l'historiographie du genre en question -, l'A. aborde successivement les deux aspects évoqués dans le sous-titre : les figures constitutives du genre satyrique, d'une part, les « configurations » opérées par la mise en intrigue des drames, d'autre part. Ces deux dimensions d'analyse doivent servir à la constitution d'une définition du drame satyrique comme genre. Ce sont les aspects figuratifs qui occupent l'essentiel du propos. Articulée en dix chapitres sur un total de douze, cette analyse aborde successivement l'espace du drame satyrique, caractérisé par sa marginalité (chap. 2), la figure intermédiaire du satyre (humain/animal; enfant/adulte; masculin/féminin; humain/divin; servilité) (chap. 3), les satyres et leur relation à la musique et à ses instruments (chap. 4), à la danse et à ses rythmes (chap. 5), à la consommation d'un vin qui prend tout son sens confronté au lait, à la bière et à l'eau (chap. 6), à la sexualité (chap. 7) et au monde des athlètes (chap. 8). Ces satyres sont également mis en scène dans des contextes de découvertes ou de résolution d'énigmes, ce qui est moins souvent souligné (chap. 9). Enfin, ce sont les figures monstrueuses et leurs caractéristiques propres (axenia, asociabilité, impiété, démesure) qui sont envisagées, surtout dans leur face à face avec les satyres (chap. 10), et enfin les figures héroïques dans le déploiement très spécifique de leur activité « satyrique », en regard des tragédies (chap. 11). Ces dix chapitres fourmillent de développements susceptibles d'intéresser l'historien de la religion grecque, dans la mesure même où l'A. revendique la nécessité de contextualiser le drame satyrique et de concevoir les réseaux dans lesquels s'insèrent ces figures comme des constructions culturelles produites par «l'ensemble des pratiques signifiantes sociales, politiques, rituelles, discursives, iconographiques - d'un groupe humain donné » (p. 33). Contre l'habitude d'aller glaner ces fragments en ordre dispersé pour servir tel ou tel propos d'histoire religieuse ou d'analyse des mythes, nous disposons désormais d'un outil qui replace chaque fragment dans un cadre plus large, et autorise donc une exploitation plus pertinente des données effectivement disponibles. C'est le cas, entre beaucoup d'autres, du célèbre fragment du drame satyrique attribué à Critias ou à Euripide et qui met dans la bouche de Sisyphe un récit sur l'invention des dieux par un homme soucieux de mettre fin aux désordres à l'outre dans le monde (43 F 19 Snell).

Le dernier chapitre, consacré aux "aspects configurationnels», tente de pallier le caractère lacunaire du corpus en identifiant un certain nombre de thèmes qui permettent de saisir les articulations et les orientations de l'action dramatique, à défaut de pouvoir en reconstituer le développement. L'A. relève cinq thèmes, que leur récurrence conduit à considérer comme génériques : 1) la neutralisation d'une figure niant les valeurs de la civilisation, 2) la production d'objets, de figures et de phénomènes revêtant un caractère prodigieux et l'expérience qu'en font les satyres, 3) les marges comme espace d'expérimentation de leurs potentialités adultes pour les enfants divins et hérö̈ques, 4) les prétentions sexuelles des satyres déjouées par l'intervention d'un dieu ou d'un héros, 5) les prétentions des satyres à assumer des rôles qui leur sont étrangers comme, par exemple, celui d'époux. Ce même chapitre thématise ensuite un fil rouge du livre, à savoir les aspects dionysiaques du drame satyrique. S'il ne se perd pas dans la question épineuse 